\title{
Study on the influencing factors and prediction of the medical cost of chronic renal failure in China based on a decision tree algorithm
}

\section{CURRENT STATUS: UNDER REVIEW}

BMC Nephrology $\triangle$ BMC Series

pingping dai

Central South University Third Xiangya Hospital

276160176@qq.comCorresponding Author

ORCiD: https://orcid.org/0000-0003-1024-7114

\section{Aijing Luo}

Central South University Xiangya School of Medicine

Weifu Chang

Key laboratory of medical information research,Third Xiangya Hospital, Central South University

Zirui Xin

Key laboratory of medical information research, Third Xiangya Hospital,Central South University

Haiwei Cheng

Department sociology, Central South University

Wei Ouyang

Key laboratory of medical information, Third Xiangya Hospital, Central South University

DOI:

10.21203/rs.2.21909/v1

\section{SUBJECT AREAS}

Urology \& Nephrology

KEYWORDS

decision tree, CHAID algorithm, CART algorithm, chronic renal failure, hospitalization costs, influencing factors, prediction 
Abstract

Purpose: The objective of this study is to explore the factors that affect the hospitalization costs for patients with chronic renal failure (CRF) and to extract relevant characteristics for modeling, so as to make predictions about hospitalization costs for CRF patients.

Methods: This study collected the data on the first page of the 2014 medical records of three firstclass tertiary hospitals in Beijing. Using IBM SPSS Modeler software, we used the chi-squared automatic interaction detector (CHAID) and classification and regression tree (CART) algorithms to construct a prediction model of the hospitalization costs for CRF patients and conducted a comparative analysis. The data of the 1819 cases in this study included the index variables on the first page of the medical records, which covered social economics, clinical characteristics, and medical consumption. The input variables included medical payment method, sex, age, marital status, length of hospital stay, main diagnosis, number of other diagnoses, major surgery, and number of surgeries. The target variable was the total medical expense.

Results: Our results showed that medical payment method, sex, age, marital status, length of hospital stay, main diagnosis, number of other diagnoses, major surgery, and number of surgeries all had an effect on hospitalization cost. There was no significant difference in the prediction models of the total hospitalization cost constructed using the CHAID and CART algorithms.

Conclusions: Major surgery and length of hospital stay were important predictor variables for modeling with both CHAID and CART algorithms. The length of hospital stay should be included in the grouping variables when disease-related grouping and prediction of medical costs are done for CRF patients.

\section{Full Text}

Due to technical limitations, full-text HTML conversion of this manuscript could not be completed. However, the manuscript can be downloaded and accessed as a PDF.

Figures 


\section{Classification rules of layer 1}

Classification rules of layer 2
Mean value of total medical expenses in each group of layer 2

\section{5 yuan}

5522.1 yuan

7040.2 yuan

8091.8 yuan

9293.1 yuan

$8<$ length of hospital stay $\leq 12$

$14,417.1$ yuan

$12<$ length of hospital stay $\leq 18$

length of hospital stay $>18$

$21,336.4$ yuan

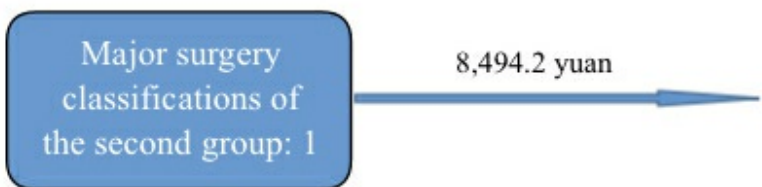

\section{length of hospital stay $\leq 18$}

length of hospital stay $>18$

\section{length of hospital stay $\leq 3$}

$3<$ length of stay $\leq 6$

$6<$ length of stay $\leq 10$

$10<$ length of stay $\leq 18$

length of hospital stay $>18$
$56,467.6$ yuan

$75,635.1$ yuan

\section{1 yuan}

7313.8 yuan

$10,587.4$ yuan

$15,816.3$ yuan

$33,451.4$ yuan

Figure 1

Calculation results of the CHAID model 


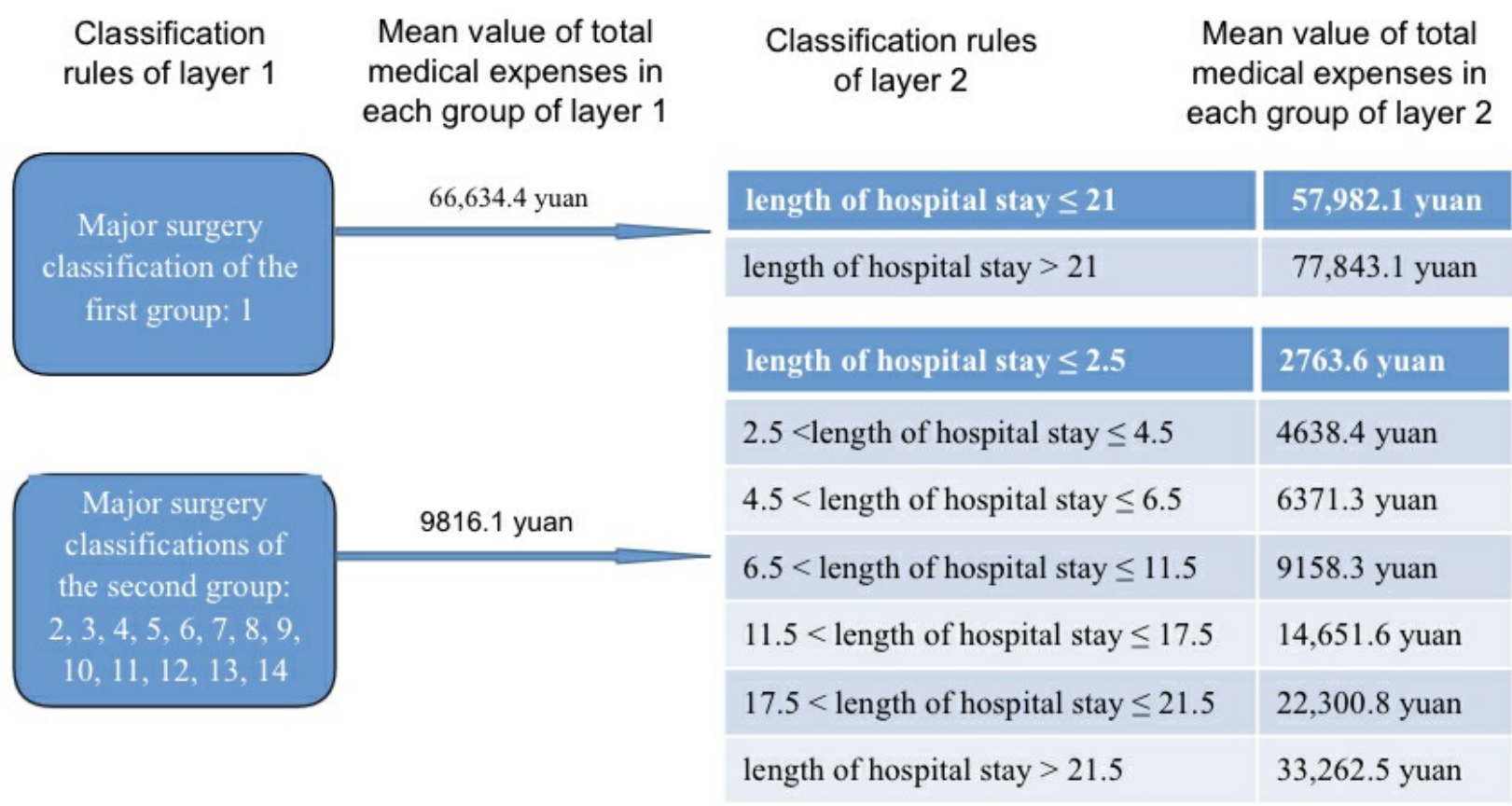

Figure 2

Calculation results of the CART model 